\title{
Pediatric use of tetracyclines: focus on neurodevelopmental effects
}

\author{
loana Inta ${ }^{1}$, Georg F. Hoffmann ${ }^{2}$ and Markus Bettendorf ${ }^{1}$
}

$T$ etracyclines represent important antibiotics that are in clinical use since many decades, particularly effective in treating infections with intracellular agents, such as mycoplasma and chlamydia. They bind with high specificity to ribosome $\mathrm{S}$ of bacteria and are considered to have a more favorable side-effect profile than other classes of antibiotics. However, accumulating evidence indicates that tetracyclines can induce several biological actions independent of their anti-microbial activity, like anti-inflammatory, anti-apoptotic, and anti-tumoral effects (1). In the brain, minocycline and doxycycline, the tetracyclines that easily cross the blood-brain barrier, were shown to effectively inhibit microglia activation associated with neuroinflammation and to provide neuroprotection in several models of neurological disorders, like cerebral ischemia, brain injury, and several neurodegenerative conditions (Parkinson's disease, Huntington's disease, amyotrophic lateral sclerosis, Alzheimer's disease, and multiple sclerosis) (1).

Of particular relevance for the pediatric use of tetracyclines are the recently reported effects on brain development. Of great interest are the results of a study using human-induced pluripotent stem cells (hiPSCs) derived from patients with Down's syndrome (DS), which revealed a major role of astrocytes in its pathogenesis (2). DS represents a severe developmental disorder caused by trisomy of the human chromosome 21, characterized by intellectual disability and impaired neurogenesis and synaptogenesis. Astrocytes derived from patients with DS had higher levels of reactive oxygen species, showed marked disturbances in the expression of several astrocytic markers, and failed to promote neurogenesis and synaptogenesis, as in the normal brain (2). In addition, astroglia play an important role also in modulating neuronal cell death in DS, which is triggered by overproduction of S100B and nitric oxide release from astrocytes. Interestingly, minocycline reverted partially the pathological phenotype of astrocytes derived from patients with DS by normalizing the expression of several markers, such as the higher levels of astroglial S100B, GFAP, and inducible nitric oxide synthase observed both in hiPSC and human brain tissue in patients with DS (2). Moreover, it prevented neuronal loss and promoted neurogenesis and neuronal maturation in hiPSC-derived DS neurons (2). The molecular and cellular mechanisms by which tetracyclines exert these neuroprotective and potentially therapeutically effective actions remain unclear. However, the neuroprotective effect of tetracyclines in DS may occur by the inflammation-independent pathways and appears to be specific for these drugs, as no beneficial effects on DS astroglia and neurogenesis were observed following the application of other effective neuroprotective compounds, such as resveratrol and curcumin (2). Future studies may clarify the unique neuroprotective potential of tertracyclines in DS.

Another recent study (3) provides mechanistic insights into beneficial effects of tetracyclines in another developmental disorder that impacts also brain functions, phenylketonuria (PKU). PKU is an inborn error of metabolism involving impaired metabolism of the amino acid phenylalanine, and is treated by a strict phenylalanine-restricted diet. Untreated, PKU can lead to progressive mental retardation. Phenylalanine forms in PKU cytotoxic amyloid-like assemblies in important brain areas such as the hippocampus and cortex, which most likely mainly contribute to the emergence of cognitive dysfunctions. Interestingly, doxycycline has the capacity to hinder amyloidogenic peptides/protein aggregation, destabilize protein aggregates, and promote their degradation: this surprising effect was discovered based on the structural similarity of tetracyclines to anthracyclines, like iododeoxyrubicine, drugs used in cancer chemotherapy, but which were reported previously to exert also an antiamyloidogenic effect. Therefore, although the underlying mechanisms of this effect are still not completely understood, it may be due to specific structural characteristics of these molecules that are most likely not related to the antimicrobial efficacy. This is of relevance not only for the potential use of tetracyclines in the treatment of neurodegenerative disorders. Interestingly, doxycycline prevents also the formation of wellordered crystalline phenylalanine assemblies and hinders their neurotoxic effects in PKU (3). Therefore, doxycycline may represent an alternative novel approach for future non-dietary preventive therapies in PKU.

${ }^{1}$ Division of Pediatric Endocrinology, Center for Pediatric and Adolescent Medicine, Heidelberg University Hospital, Heidelberg, Germany; ${ }^{2}$ Pediatric Neurology and Metabolic Medicine, Center for Rare Disorders, Center for Pediatric and Adolescent Medicine, Heidelberg University Hospital, Heidelberg, Germany. Correspondence: loana Inta (loana.Inta@med.uni-heidelberg.de)

Received 9 May 2017; accepted 20 June 2017; advance online publication 2 August 2017. doi:10.1038/pr.2017.167 


\section{Commentary | Inta et al.}

Altogether, these studies, corroborated with the neuroprotective effects of tetracyclines at adult stages, suggest valuable therapeutic actions both in the young and adult brain. Therefore we aimed to test whether minocycline protects also against apoptosis triggered in the mouse brain by the NMDAR antagonist MK-801, that is known to induce extensive neurotoxicity at early postnatal stages, corresponding to the so-called brain growth spurt period in which the brain grows at an accelerated rate (4). Surprisingly, instead of eliciting neuroprotective effects, minocycline induced a massive and widespread cortical apoptosis and exacerbated MK-801-triggered cell death (4). Moreover, in some areas like the subiculum the pro-apoptotic effect of minocycline was even more pronounced than that elicited by MK-801 (ref. 4). This result is in agreement with the previous data showing that minocycline induces marked neuronal cell death in the early postnatal somatosensory cortex by severely disturbing the distribution, morphology, and functional properties of microglia cells (5). The mechanisms of minocycline-induced neurotoxicity are also not known. One proposed explanatory model is based on the inhibitory effect of minocycline on matrix metalloproteinases, which play a critical role in regulating the production of growth factors and are progressively downregulated during the first postnatal week (5). Nevertheless, also deleterious effects of inhibiting microglia activation during a critical developmental window in contributing to these neurotoxic effects cannot be excluded.

What could be the significance and long-term consequences of these neurotoxic actions of tetracyclines in the immature brain? Up to date, there are no studies regarding the protracted behavioral effects of tetracycline use. However, common anesthetic agents with antagonistic action on glutamate NMDA receptors induced persistent memory/ learning impairments at adult stages following early life exposure to these substances. Such data, although obtained in animal models and limited to a relatively narrow time window, may raise serious concerns regarding the common pediatric use of these tetracyclines.

In sum, there is strong support for the idea that brainpenetrant tetracyclines induce a variety of important effects during brain development, ranging from potential therapeutic applications to age-dependent massive neurotoxic effects (Figure 1). Although the basic mechanisms of these effects yet poorly understood, most likely they are not related to the classical use as antimicrobial agents. Whereas novel studies provide exciting hints about their possible benefit in pediatric disorders with an important neurodevelopmental component,

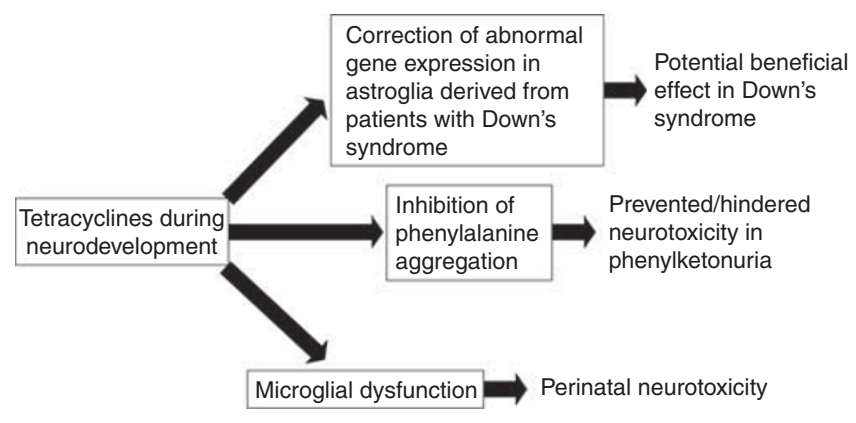

Figure 1. Schematic representation of potential beneficial effects of tetracyclines in neurodevelopmental disorders, like DS and PKU, but also of neurotoxic effects (see refs $2-5$ ).

like DS and PKU, there are also reasons to be cautious regarding their deleterious effects on brain development. Hereby, one main conclusion of research in the last years is that microglia activation is not only a phenomenon that accompanies pathological processes (neuroinflammation), but that represents a physiological process of critical importance during neurodevelopment. Therefore, its inhibition by minocycline or doxycycline may also seriously disturb normal brain development. More research is needed to better understand the mechanisms of action of these tetracyclines in the young brain for clarifying their effects and side-effects.

\section{STATEMENT OF FINANCIAL SUPPORT}

This work was supported by an Olympia-Morata grant and the Innovation Fund Frontier of the University of Heidelberg as well as by the Brigitte Schlieben Lange Programme of the state of Baden-Württemberg to I.I.

Disclosure: The authors declare no conflict of interest.

\section{REFERENCES}

1. Garrido-Mesa N, Zarzuelo A, Gálvez J. Minocycline: far beyond an antibiotic. Br J Pharmacol 2013;169:337-52.

2. Chen C, Jiang P, Xue H, et al. Role of astroglia in Down's syndrome revealed by patient-derived human-induced pluripotent stem cells. Nat Commun 2014;5:4430.

3. De Luigi A, Mariani A, De Paola $M$, et al. Doxycycline hinders phenylalanine fibril assemblies revealing a potential novel therapeutic approach in phenylketonuria. Sci Rep 2015;5:15902.

4. Inta I, Vogt MA, Vogel AS, et al. Minocycline exacerbates apoptotic neurodegeneration induced by the NMDA receptor antagonist MK-801 in the early postnatal mouse brain. Eur Arch Psychiatry Clin Neurosci 2016;266:673-7.

5. Arnoux I, Hoshiko M, Sanz Diez A, et al. Paradoxical effects of minocycline in the developing mouse somatosensory cortex. Glia 2014;62: $399-410$. 